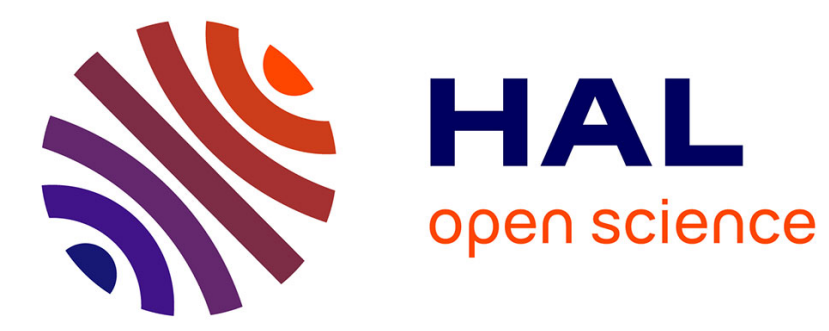

\title{
Predictions of Taylor's power law, density dependence and pink noise from a neutrally modelled time series
}

Petr Keil, T. Tomáš Herben, James Rosindell, David Storch

\section{To cite this version:}

Petr Keil, T. Tomáš Herben, James Rosindell, David Storch. Predictions of Taylor's power law, density dependence and pink noise from a neutrally modelled time series. Journal of Theoretical Biology, 2010, 265 (1), pp.78. 10.1016/j.jtbi.2010.04.014 . hal-00598093

\section{HAL Id: hal-00598093 https://hal.science/hal-00598093}

Submitted on 4 Jun 2011

HAL is a multi-disciplinary open access archive for the deposit and dissemination of scientific research documents, whether they are published or not. The documents may come from teaching and research institutions in France or abroad, or from public or private research centers.
L'archive ouverte pluridisciplinaire $\mathbf{H A L}$, est destinée au dépôt et à la diffusion de documents scientifiques de niveau recherche, publiés ou non, émanant des établissements d'enseignement et de recherche français ou étrangers, des laboratoires publics ou privés. 


\section{Author's Accepted Manuscript}

Predictions of Taylor's power law, density dependence and pink noise from a neutrally modelled time series

Petr Keil, Tomáš Herben, James Rosindell, David Storch

PII: S0022-5193(10)00197-9

DOI: doi:10.1016/j.jtbi.2010.04.014

Reference: YJTBI 5963

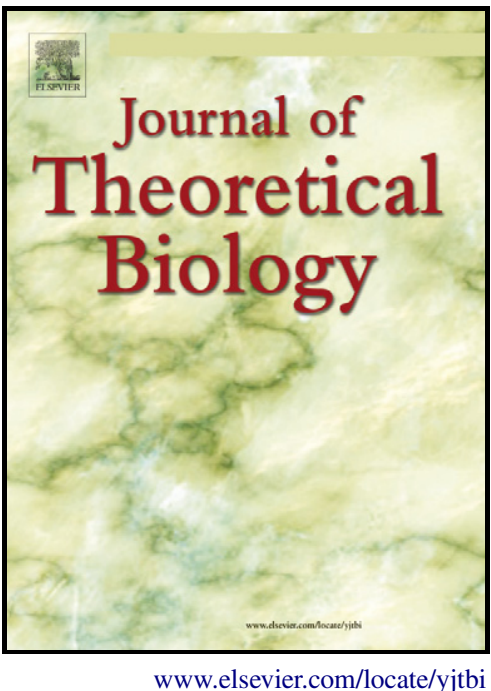

To appear in: $\quad$ Journal of Theoretical Biology

Received date: 16 December 2009

Revised date: $\quad 12$ April 2010

Accepted date: 12 April 2010

Cite this article as: Petr Keil, Tomáš Herben, James Rosindell and David Storch, Predictions of Taylor's power law, density dependence and pink noise from a neutrally modelled time series, Journal of Theoretical Biology, doi:10.1016/j.jtbi.2010.04.014

This is a PDF file of an unedited manuscript that has been accepted for publication. As a service to our customers we are providing this early version of the manuscript. The manuscript will undergo copyediting, typesetting, and review of the resulting galley proof before it is published in its final citable form. Please note that during the production process errors may be discovered which could affect the content, and all legal disclaimers that apply to the journal pertain. 
1 Predictions of Taylor's power law, density dependence and pink

2 noise from a neutrally modelled time series

3

4 Authors: Petr Keil ${ }^{1, *}$, Tomáš Herben ${ }^{2,3}$, James Rosindell ${ }^{4} \&$ David Storch ${ }^{1,5}$

5

$6 \quad{ }^{1}$ Department of Ecology, Faculty of Science, Charles University, Vinicna 7, 12800

$7 \quad$ Praha 2,Czech Republic,e-mail:pkeil@seznam.cz

8 tel: $00420-776371840$

$9{ }^{2}$ Institute of Botany, Academy of Sciences of the Czech Republic, Prùhonice, 252 43,

10 Czech Republic, e-mail: herben@site.cas.cz

$11{ }^{3}$ Department of Botany, Faculty of Science, Charles University, Benátská 2, Praha 2,

12128 01, Czech Republic

$13{ }^{4}$ Institute of Integrative and Comparative Biology, Faculty of Biological Sciences,

14 University of Leeds, Leeds LS2 9JT, United Kingdom, e-mail:

$15 \quad$ j.l.rosindell@leeds.ac.uk

$16{ }^{5}$ Center for Theoretical Study, Charles University in Prague and the Academy of

17 Sciences of the Czech Republic, Jilská 1, 11000 Praha 1, Czech Republic, e-mail:

18 storch@.cts.cuni.cz

19

$20 *$ Corresponding author

21 


\section{Abstract}

23 There has recently been increasing interest in neutral models of biodiversity and their

24 ability to reproduce the patterns observed in nature, such as species abundance

25 distributions. Here we investigate the ability of a neutral model to predict phenomena

26 observed in single-population time series, a study complementary to most existing

27 work that concentrates on snapshots in time of the whole community. We consider

28 tests for density dependence, the dominant frequencies of population fluctuation

29 (spectral density) and a relationship between the mean and variance of a fluctuating

30 population (Taylor's power law). We simulated an archipelago model of a set of

31 interconnected local communities with variable mortality rate, migration rate,

32 speciation rate, size of local community and number of local communities. Our

33 spectral analysis showed 'pink noise': a departure from a standard random walk

34 dynamics in favor of the higher frequency fluctuations which is partly consistent with

35 empirical data. We detected density dependence in local community time series but

36 not in metacommunity time series. The slope of the Taylor's power law in the model

37 was similar to the slopes observed in natural populations, but the fit to the power law

38 was worse. Our observations of pink noise and density dependence can be attributed

39 to the presence of an upper limit to community sizes and to the effect of migration

40 which distorts temporal autocorrelation in local time series. We conclude that some of

41 the phenomena observed in natural time series can emerge from neutral processes, as

42 a result of random zero-sum birth, death and migration. This suggests the neutral

43 model would be a parsimonious null model for future studies of time series data. 
45 Key words: random walk, spectral analysis, mean-variance scaling, stochasticity, neutral theory of biodiversity, autocorrelation, 1/f noise

\section{Introduction}

51 The neutral theory of biodiversity (NTB; Hubbell, 2001; Chave, 2004; Alonso et al., 52 2006; Leigh, 2007; Rosindell et al., 2010) has revolutionized the way we think about

53 species coexistence over the last decade. The assumptions of the theory are

54 controversial (Tilman, 2004; Leibold and McPeek, 2006) and some argue that there is 55 limited empirical support (McGill et al., 2006), but the theory still has huge potential as a null model (Gotelli and McGill, 2006), serving as an essential first approximation

57 to reality and a base for more complex theories. Even with its apparently

58 oversimplified set of assumptions, the NTB is capable of predicting realistic species-

59 abundance distributions (Hubbell, 2001; Etienne, 2005) and species-area curves

60 (Rosindell and Cornell, 2007, 2009), as well as many other ecological patterns

61 (Herben, 2004, 2009). Most of the current research is focused on the community-level

62 predictions of a single snapshot in time at equilibrium (Chave et al., 2002; McGill,

63 2003; Turnbull et al., 2005; Volkov et al., 2005; Dornelas, et al. 2006). Dynamical

64 behaviour of populations in NTB has rarely been studied. Azaele et al. (2006) used

65 ratios of population sizes between two snapshots of time to estimate species turnover

66 rates and extinction times of trees in Barro Colorado Island, Panama. Mutshinda et al.

67 (2008) produced the only study (as far as we are aware) that examines sufficiently

68 long real-world time series from the perspective of NTB. They used the Bayesian

69 statistical framework and a neutral model of community dynamics (zero-sum 
assumption relaxed) to show that sizes of natural communities fluctuate more than expected under neutrality. However, Mutshinda et al. (2008) focused only on the dynamics of local community size and they did not examine time series of individual species.

There are some characteristics of natural time-series that are often used as summary statistics and can be employed here in making comparisons with the time series produced by a neutral model. For example, natural time series have been shown to obey some degree of stabilizing density dependence (Woiwod and Hanski, 1992; Brook and Bradshaw, 2006; Sibly et al., 2007). Natural populations also follow a specific relationship between mean population size $(m)$ and variance of population size $\left(s^{2}\right)$ called Taylor's Power Law (TPL) (Taylor et al., 1983; Tokeshi, 1995; Ballantyne and Kerkhoff, 2007) which has the form $s^{2} \propto m^{b}$. In natural populations, the exponent $b$ of TPL is usually observed to satisfy $1<b<2$ (Kendal, 2004). Natural time series can be considered as being the net result of a number of independent fluctuations each with a distinct frequency and amplitude. The amplitude (or 'spectral density') $S(f)$ is a function of the frequency $f$ and often satisfies $S(f) \propto 1 / f^{\gamma}$ (Halley, 1996). The exponent $\gamma$ usually falls between 0 and 2 for natural time series (Arino and Pimm, 1995; Halley, 1996; Inchausti and Halley, 2001, 2002), a property known as 'pink noise'.

The null model for discrete population dynamics is an unbounded random

90 walk of population size $(N)$ and is described as

$$
\ln N_{t+1}=\ln N_{t}+e_{t}
$$

93 is independent on population size (Murdoch, 1994; Hanski et al., 1996). The model is

94 a discrete first-order Markov chain in which all the information that can influence the 
future state $(t+1)$ is fully captured in the present state $(\mathrm{t})$. Random walk time series are often termed "brownian" or autocorrelated noise. Random walk is synonymous for a completely density-independent process in ecology, it produces a slope of $b \approx 2$ in Taylor's power law and spectral scaling exponent of $\gamma \approx 2$.

Although the term random walk is sometimes used to describe the population

100 time series in neutral models (Hubbell, 2001; Alonso et al., 2006), there are reasons

101 why neutral models can behave differently from the random walk as defined above.

102 Firstly, there is usually a constraint on the total number of individuals (the 'zero-sum'

103 rule) which imposes a clear upper limit to any population fluctuations (Hubbell, 2001;

104 Hubbell and Lake, 2003). Secondly, immigration of individuals into the local

105 community can cause a locally extinct species to reappear (zero is not necessarily

106 absorbing; Hubbell, 2001) and finally immigration can distort the temporal

107 autocorrelation of $N$, (the number of individuals belonging to the species of interest in

108 a local community). In the presence of immigration, $N_{t+1}$ for a given local community

109 does not depend solely on $N_{t}$ for that community (as in the autocorrelated random

110 walk), it also depends on the $N_{t}$ for all the other local communities (the

111 metacommunity).

112 In this paper we ask if a simple neutral model can produce time series that are

113 similar to natural time series. In particular, we show that populations in the neutral

114 model can produce realistic slopes of the Taylor's power law, have spectral properties

115 similar to natural populations and seem to reveal density-dependency more frequently

116 than expected in a random walk. Additionally, we explore the influence of the NTB

117 parameters (size of local communities, number of local communities, mortality rate,

118 migration and mutation rate) on these properties of population time series. 
120 Methods

122 The simulation model

123 Instead of the classic neutral model used by Hubbell (2001) and Volkov et al. (2003),

124 we simulate a metacommunity consisting of an archipelago of $L$ isolated local

125 communities interconnected by migration at rate $m$ (see Fig. 1 below and Volkov et

126 al., 2007). Each local community contains $J$ individuals all of which behave in an

127 identical manner irrespective of the species to which they belong. The archipelago

128 model enabled us to obtain data on abundances and their variances for one species at

129 multiple local communities, which is necessary for the analysis of Taylor's Power

130 Law. We used the simulation approach because it enabled us to record the species

131 identity of every individual at each time step and across all local communities.

132 Furthermore, we wished to collect time series of limited lengths that are comparable

133 to those of empirically collected time series in order to accurately mimic any sampling

134 effects present in empirical data. This would be particularly difficult to approach

135 analytically. Our simulations were conducted using a 'forwards in time' approach. A

136 'backwards in time' or coalescence approach can be much faster (Rosindell et al.,

137 2008), but the main strengths of this approach are only present when collecting a snap

138 shot in time of a sample from a very large or infinite metacommunity. We are instead

139 collecting single species time series data and sampling all individuals from a

140 relatively small metacommunity: a scenario where coalescence is far less beneficial.

141 Four processes operate in the local community at each step: mortality $(d$;

$1420<d<1)$, local replacement $(l ; 0<l<1)$, immigration from outside of the local

143 community $(m ; m=1-l)$ and speciation $(s ; 0<s<1)$ in that order (Fig. 1, Table 1).

144 To model mortality, $J \times d$ individuals were randomly removed from each local 
community in every time step with all individuals having an equal probability of removal. These removed individuals were then replaced by offspring from others in the local community and other local communities (immigration). Local replacement was modeled by drawing individuals to reproduce at random from the $J(1-d)$ individuals that survived mortality until $J \times d \times l$ offspring have been created. The remaining $J \times d \times m$ dead individuals were replaced by immigration from all of the other local communities. The probability of immigration of a species is given by its relative abundance in the whole metacommunity (excluding the local community in focus).

A local population can occasionally give rise to a new species (a speciation event), this happens with a fixed probability at each time step for each local population of a species. When speciation occurs, individuals of the given species in the local community were randomly split into two parts, one of which forms the initial abundance of a new species (a version of random-fission model; Hubbell, 2001; Etienne and Haegeman, 2010; Haegeman and Etienne, 2010). Pilot testing of a point mutation model (Hubbell, 2001) showed that the choice of a speciation model does not affect the outcome of the simulations, this is not entirely surprising because for a single species population time series, speciation can be regarded as another form of mortality: individuals belonging to our focal species that switch to a new species reduce the population of our focal species in the same way as dead individuals. Each simulation was launched with only one species that occurred in all local communities and was left running for 5000 steps which we found was always sufficient for the system to reach a steady state. The last 100 steps of the simulation were then taken and used for analyses. 


\section{Spectral analysis}

We performed 200 simulations for each combination of model parameters (Table 1). From each simulation we randomly selected one species and from this species we used two time series: (1) time series of population sizes within a randomly selected local community and (2) time series of population sizes within the whole metacommunity. In cases where the species went extinct in one or both time series we randomly selected another one thereby conditioning the time series as being drawn from a locally extant species. For these time series we conducted spectral analysis (Halley, 1996; pages 680-681 in Legendre and Legendre, 2003) and estimated the value of the exponent $\gamma$ in $S(f) \propto 1 / f^{\gamma}$ where $S(f)$ is spectral density at given frequency $f\left(0 \leq f \leq f_{n / 2} ; n\right.$ is length of the time series). Values of $S(f)$ were obtained through Fast Fourier Transform of the log-transformed time series data. The spectral exponent $\gamma$ was estimated as minus the regression slope of the $\log (S(f))$ versus $\log (f)$. This procedure is exactly the same as used by Inchausti and Halley (2001) and hence our estimates of $\gamma$ are directly comparable to theirs (Fig. 2). The value of $\gamma$ can vary from $\gamma<0$ ('blue noise') through $\gamma=0$ ('white noise'), $\gamma \approx 1$ ('pink noise') and $\gamma=2$ ('brown noise' or random walk) to $\gamma>2$ ('black noise'). We use the term 'whitening' of the spectra for a deviation of $\gamma$ from 2 towards 0.

To assess effects of the neutral model parameters on $\gamma$ we fitted a multiple ANOVA model with $\gamma$ as the response variable and five explanatory variables: mortality rate, migration rate, speciation rate, number of local communities and number of individuals. Since we were dealing with simulated data we only report $R^{2} \mathrm{~s}$ and do not report any $P$-values because these could be modified arbitrarily by changing number of simulations. Comparisons were made with the values of $\gamma$ measured in natural populations taken from the Global Population Dynamics 
195 Database (kindly provided by Pablo Inchausti; see also Inchausti and Halley, 2001,

196 2002).

197

198 Testing for density dependence

199 Using the same data, we conducted three distinct tests for density dependence in the

200 time series: Pollard's randomization test (Pollard et al., 1987), Dennis and Taper's

201 parametric bootstrap maximum likelihood test (PLBR; Dennis and Taper, 1994) and

202 Bulmer's test (Bulmer, 1974). The Pollards's test is a distribution-free likelihood ratio

203 randomization test in which a random walk (Eq. 1) or a random walk with trend are

204 density independent hypotheses, whereas the density dependent hypothesis is defined

205 as a first-order linear autoregression model (stochastic Gompertz model):

$\ln N_{t+1}=\ln N_{t}+r+\beta \ln N_{t}+e_{t} \quad(\beta \neq 1)$

where $r$ and $\beta$ are model parameters. The Bulmer's test is a parametric

208 predecessor of the Pollard's test and has been criticized for weak performance in cases

209 where there was a trend in the data $(r \neq 0)$. The PLBR test is a likelihood ratio test

210 which uses the first-order non-linear autoregression model (stochastic logistic model)

211 as the density dependent hypothesis:

$$
\ln N_{t+1}=\ln N_{t}+r+\beta N_{t}+e_{t}
$$

213 All of these tests have been criticized for disputable performance when there is

214 a census error affecting the estimation of population abundance in the field (Shenk et

215 al., 1998; Freckleton et al., 2006), but this is not the case for our simulated data. All

216 three tests gave nearly identical results and we therefore only present the output from

217 Pollard's randomization test (Pollard et al., 1987) which is 'Pollard's $P$ ': the

218 probability of observing the empirical data in the case of the density independent

219 hypothesis (Eq. 1). Following the approach of Woiwod and Hanski (1992) we 
220 normalized the distribution of Pollard's $P$ by logit transformation. To assess effects of

221 the neutral model parameters on Pollard's $P$ we again fitted a multiple ANOVA

222 model in which we used the values of logit-transformed Pollard's $P$ as the response

223 variable.

\section{Taylor's power law}

226 To test if populations in our model obey the Taylor's Power Law (TPL) we performed

22750 simulations for each combination of parameters (Table 1). For each species in the 228 simulations we created a metapopulation matrix (MPM) of abundances in which rows

229 represent the last 100 time steps of the simulation and columns represent the

230 populations in different local communities. We excluded all MPMs in which the

231 species had gone globally extinct. We studied the TPL in its temporal context (Taylor

232 and Woiwod, 1982) which means that variances and means are calculated over each

233 column (one column is one local population) in the MPM - when plotted, each point

234 in the log-log plot therefore represents one local population. Using rows instead of

235 columns in the MPM would yield the TPL in a spatial context, but the temporal

236 context is more germane to this time series study. A linear regression was fitted in

237 each of the log-log plots and the TPL slope (parameter $b$; Taylor, 1961; Tokeshi,

238 1995) and the coefficients of determination $\left(R^{2}\right)$ were calculated. As with our other

239 results, the simulated values of TPL slopes and $R^{2}$ s were compared with empirically

240 observed values (taken from Taylor and Woiwod, 1980, 1982).

\section{Results}

\section{Spectral analysis}


245 Values of the scaling exponent $\gamma$ estimated from our simulations are summarized in

246 Figure 2. For local communities, the median value of $\gamma$ was $1.59(2.5 \%$ and $97.5 \%$

247 quantiles were 1.26 and 2.19 respectively). The value of $\gamma$ was only influenced by

248 mortality rate and migration rate (Table 2, Fig. 3) with higher mortality and higher

249 migration each producing time series that were closer to pink noise $(\gamma<2)$ (Fig. 2).

250 On the metacommunity scale, the median of $\gamma$ was $1.75(2.5 \%$ and $97.5 \%$ quantiles

251 were 1.05 and 2.07 respectively). We did not detect any influence of the model

252 parameters on the value of $\gamma$ at the metacommunity scale (Table 2, Fig. 3). Our

253 simulations produced $\gamma$ values falling within a narrower interval than is generally

254 observed in nature (Fig. 2), however, they departed from 2 (the value for an

255 unbounded random walk) in the same direction as for natural populations.

256 In general, the population time series emerging from our neutral model had

257 lower $\gamma$ than would be expected for an autocorrelated random walk $(\gamma=2)$. This

258 could partly be an artifact caused by the insufficient length of time series. It was

259 recently shown that random walks of length $\sim 100$ actually give estimates of between

$260 \quad 1.5$ and 1.8 instead of 2 because of the missing low-frequency fluctuations

261 (Miramontes and Rohani, 2002). Nevertheless, since empirically collected time series

262 are rarely longer than 100 sequential observations, our simulations of time series of

263 similar lengths are amply justified to purposefully include the effects of sampling over

264 a limited time scale. Since we also showed that the vales of $\gamma$ tended to be lower at

265 local scales when comparing time series of equal lengths, we can still conclude that

266 some whitening of spectra at local community sales is caused by more than just the

267 length of the sampled time series. 
270 On the scale of local communities the median of Pollard's $P$ was $0.14(2.5 \%$ and

$27197.5 \%$ quantiles were 0 and 0.82 respectively) indicating a distribution skewed

272 towards the lower values of Pollard's $P$. We also found that the values of Pollard's $P$

273 were dependent on mortality rate and migration rate (Table 2, Fig. 4). Similarly to

274 spectral analysis, simulations with higher mortality rate and higher migration rate

275 produced time series with lower values of Pollard's $P$ (Fig. 4). On the

276 metacommunity scale the median value of Pollard's $P$ was $0.5(2.5 \%$ and $97.5 \%$

277 quantiles being 0.01 and 0.97 respectively). This indicates no clear signal of density

278 dependence on the metacommunity scale. We did not detect any influence of the other

279 model parameters on the values of logit-transformed Pollard's $P$ (Table 2, Fig. 4).

280 Pollard's $P$ varied with relative abundance of species in local communities but not in 281 metacommunities (Fig. 6).

\section{Taylor's power law (TPL)}

284 The median slope of TPL was 1.73 (2.5\% and $97.5 \%$ quantiles were 0.059 and 2.49

285 respectively). The coefficients of determination $\left(R^{2}\right)$ of power law regressions varied,

286 following skewed, non-normal distributions (Figs. 2, 5), having generally lower

287 values to what has been observed in nature (Fig. 2). The effects of model parameters

288 on the slope of TPL was not as distinct as in spectral analysis and density dependence

289 analysis; we detected a weak influence of number of local communities and mortality

290 rate on the slopes of TPL (Table 2, Fig. 5). Both the slopes and $R^{2}$ s showed negative

291 dependence on the mean relative abundance of species (Fig. 7).

\section{Discussion}


296 The observed whitening of spectra in our model is not what we would expect under random walk dynamics. One possible reason for this is the effect of migration which

298 can cause the spectral whitening. A population in an isolated local community (low or

299 no immigration from outside) follows dynamics similar to an autocorrelated random

300 walk $(\gamma \approx 2)$ in which there is little or no dependence on the abundances in other local

301 communities. However, after introducing immigration from outside of the local

302 community, the autocorrelative nature of the process is broken and the species

303 abundance in the whole metacommunity becomes important. The influence of single

304 local community dynamics on the metacommunity are likely to be small (inversely

305 proportional to the number of local communities), but in contrast the influence of the

306 metacommunity on local community dynamics can be substantial (proportional to the

307 migration rate). Hence, the migration causes the local time series to be whitened (non-

308 autocorrelated in time) while the metacommunity time series remains "brownian"

309 (autocorrelated in time). The mortality rate can also cause spectral whitening by

310 adding random population fluctuations at high frequencies, but not at low ones

311 subsequently lowering $\gamma$. We expect that this effect of mortality rate would not be

312 apparent in very long time series (> 400 time steps; Miramontes and Rohani, 2002).

313 However, such long time series are nearly never available in ecology and we wanted

314 to keep our time series comparably long to the real-world ones.

315 The spectral analysis results are mirrored by the results of the tests for density

316 dependence. Density dependence is modeled either as stochastic Gompertz (Eq. 2) or

317 stochastic logistic (Eq. 3) models in the tests we used (Shenk et al., 1998, see also

318 Methods). 
It has been recognized for a long time (Eberhardt, 1970; Freckleton et al.,

320 2006) that a sequence of uncorrelated random numbers ("white noise") tends to

321 follow a pattern consistent with density dependence. If you take white noise time series and plot changes of $\ln$ population size $\left(\ln N_{t+1}-\ln N_{t}\right)$ against $\ln N_{t}$ (where $N_{t}$ is the number of individuals at time t) you will get a linear negative relationship, corresponding (spuriously) to the density dependent Gompertz model (Eq. 2) with $\beta \approx-1$. Moreover, white noise can be very similar to the output of the stochastic logistic model (Eq. 3) with $\beta<0$. We have learned that the whitening of a spectra

327 means bringing it from $\gamma \approx 2$ down towards 0 , which is white noise. We also know

328 that migration (by breaking the autocorrelation structure) and mortality rate (by

329 increasing spectral density at high frequencies in time series of limited length) cause

330 spectral whitening. The detection of density dependence can also be attributed to the same mechanisms, although most ecologists would consider such density dependence

332 to be spurious (Freckleton et al., 2006).

Ucorrelated noise in the form of census error also causes spurious detection of

334 density dependence has long been known to population ecologists (Shenk et al., 1998;

335 Freckleton et al., 2006). Moreover, Freckleton et al. (2006) showed an example of a population that follows a random walk but consists of two subpopulations each of

337 which apparently shows density dependence because there is random migration

338 between them. That immigration can cause time series to resemble a sequence of 339 uncorrelated random numbers was also noted by Anderson et al. (1982) and this is 340 indeed the case for our neutral model. There should also be real density dependence in our model. In an isolated

342 community, the closer $N_{t}$ gets to zero (extinction) or to $J$ (monodominance) the lower 343 the probability of $N_{t+1}$ differing from $N_{t}$ will be. When a species almost fills the local 
344 community, its probability of increasing its abundance in the next time step is small,

345 because it is unlikely that one of the few individuals belonging to a different species

346 will be selected for mortality. Similarly, when the abundance of a species is low, its

347 probability of reproducing is low because after mortality it is not likely to be selected

348 to reproduce. The highest rate of fluctuations and lowest incidence of density

349 dependence is then expected when a population has a relative abundance of 0.5

350 (Hubbell, 2001). We detected this form of density dependence only in local

351 communities at low relative abundances (Fig. 6). Species with high relative

352 abundances were rare in our simulations and hence we were unable to clearly show

353 the real density dependence for them (Fig. 6) even though in theory it must exist.

355 Taylor's power law (TPL)

356 The archipelago neutral model produced slopes of Taylor's Power Law with median

357 close to the range observed in natural populations $(1<b<2)$, but with higher incidence

358 of low coefficients of determination. In order to explain this we need to summarize

359 the mean-variance scaling properties of simple stochastic models. If a species

360 undergoes an unrestricted random walk (Eq. 1), the TPL has a slope $b=1$ at very low

361 population densities and gradually changes to $b=2$ at a certain density (Anderson et

362 al., 1982; Tokeshi, 1995; Keeling, 2000). The population density at which $b$ starts to

363 change from 1 to 2 depends on the relative magnitude of stochastic changes because

364 of the high incidence of extinctions at low population densities which lower the

365 variance. This effect can explain $b<2$ at very low densities.

366 Hubbell (2001) shows what happens with the mean-variance scaling if the

367 stochastic population fluctuations are restricted by an upper boundary (the zero-sum

368 rule) and are fed by immigration, although Hubbell does not use the term Taylor's 
power law. Hubbell (2001) predicts hump-shaped parabolic relationship between abundance of species in a metacommunity and the variance of a local population size undergoing the zero-sum ecological drift. He also hypothesizes that most of the

372 species in nature have low relative abundances and hence should occur in the left part

373 (relative abundance $<0.2$ ) of the parabola which is nearly linear in the log-log space,

374 resembling the power-law with slope of 2. Although Hubbell's (2001) reasoning

375 focuses on the relationship between mean and variance in a set of species within one

376 community (one point in the mean-variance plot represents one species) it can apply

377 to the set of single-species local populations in our model: the mean relative

378 abundances of species in local communities were rarely higher than 0.2 in our

379 simulations and indeed, there is a much better fit of the TPL for species with low

380 abundances (Fig. 7). The poor fit at higher relative abundances is then a result of

381 fitting the linear TPL on the actually parabolic mean-variance relationship.

382 Consequently, as the mean relative abundance of species increases, the slope of the

383 TPL decreases (Fig. 7).

\section{Comparison with empirical data and conclusions}

386 Our model produces qualitatively similar patterns to those observed in nature. This is

387 perhaps surprising given the assumptions made by the neutral model, but it does again

388 highlight its use as a powerful null model.

389 There is a general agreement that natural time series are not "brownian" (Fig.

390 1; Arino and Pimm, 1995; Inchausti and Halley, 2001, 2002) - they clearly fall into

391 the whitened range of scaling exponents $(0<\gamma<2$; Fig. 2). Our simulations indicate

392 that neutral models have the potential to explain part of this observed whitening,

393 although this potential may be limited. Although higher migration rates can result in 
394 time series with more realistic values of $\gamma$, our migration rates are similar $(10 \%)$ or

395 much higher $(30 \%)$ than what is usually observed in natural metacommunities (see

396 Volkov et al., 2003 and Mutshinda et al., 2008). Mutshinda et al. (2008) already

397 showed that sizes of local communities fluctuate more than is expected from neutral

398 theory. This resonates with our simulations of individual species time series which

399 show rather high values of $\gamma$ (i.e. lower importance of high-frequency fluctuations)

400 when compared to empirical data. Recent progress in population ecology suggests that

401 a promising explanation for these high fluctuations of natural populations might be

402 environmental stochasticity (Lande et al., 2006; Mutshinda et al., 2009) which is

403 absent in neutral models, but not impossible to include in future and more general

404 versions of the original theory (Allen and Savage, 2007).

405 Realistic slopes of TPL can be produced by a number of different mechanisms

406 (Tokeshi, 1995; Keeling, 2000; Kendal, 2004; Ballantyne and Kerkhoff, 2007) and it

407 is therefore difficult to infer any definitive underlying mechanisms from its shape

408 (Mitzenmacher, 2003). Here we add neutral models to the list of possible mechanisms

409 that can produce realistic TPL slopes, although not necessarily realistic fits.

410 In conclusion, our results show that realistic slopes of Taylor's power law and

411 density dependence can emerge from a simple neutral model, whilst spectral colours

412 are less likely, but still possible to be reproduced by the neutral model. Still some

413 other natural phenomena remain unexplained by our model, such as the possibility for

414 very small $\gamma$ closer to white noise. Our findings suggest the interesting possibility that

415 the properties of population time series observed in nature are in part due to simple

416 factors such as sampling effects and dispersal limitation, which would then represent

417 the most parsimonious explanation of observed patterns. The patterns of population

418 fluctuations observed in nature may not reflect resource-driven species-specific 
419 dynamics but instead processes at the metacommunity scale, with an important effect

420 of stochastic mortality and random immigration of individuals into local communities.

421 The neutral model encompasses these factors without being overly complex and we

422 therefore suggest that neutral models make more powerful null models for time series

423 data compared to alternatives such as Brownian random walks.

\section{Acknowledgements}

427 We are grateful to Rampal Etienne, Stephen Cornell, William E. Kunin and Samuel

428 Zajicek for discussions on the subject and to one anonymous referee for helpful

429 comments. Pablo Inchausti generously provided us the GPPD data on spectral

430 exponents. The study was supported by the EU FP7 project SCALES (project

431 226852), by the Grant Agency of Charles University (GAUK 106108), by the Grant

432 Agency of the Academy of Sciences of the CR (IAA601970801) and by the Czech

433 Ministry of Education (LC06073, MSM0021620845). James Rosindell was supported

434 with funding from the EPSRC (grant EP/F043112/1). Petr Keil was supported with

435 Marie Curie Early Stage Training fellowship at University of Leeds (UK).

437 References

439 Allen, A.P., Savage, V.M. 2007. Setting the absolute tempo of biodiversity dynamics. Ecol. Lett. 10, 637-646.

441 Alonso, D., Etienne, R.S., McKane, A.J. 2006. The merits of neutral theory. Trends

442 Ecol. Evol. 21, 451-457., doi:10.1016/j.tree.2006.03.019. 
Anderson, R.M., Gordon, D.M., Crawley, M.J., Hassell, M.P. 1982. Variability in the abundance of animal and plant species. Nature 296, 245-296.

Arino, A., Pimm, S.L. 1995. On the nature of population extremes. Evol. Ecol. 9, 429443.

Azaele, S., Pigolotti, S., Banavar, J.R., Maritan, A. 2006. Dynamical evolution of ecosystems. Nature 444, 926-928.

Ballantyne, F., Kerkhoff, A.J. 2007. The observed range for temporal mean-variance scaling exponents can be explained by reproductive correlation. Oikos 116, 174-180.

Brook, B.W., Bradshaw, C.J.A. 2006. Strength of evidence for density dependence in abundance time series of 1198 species. Ecology 87, 1445-1451.

Bulmer, M.G. 1974. A statistical analysis of the 10-year cycle in Canada. J. Anim. Ecol. 43, 701-718.

Chave, J. 2004. Neutral theory and community ecology. Ecol. Lett. 7, 241-253.

Dennis, B., Taper, M.L. 1994. Density dependence in time series observations of natural populations: Estimation and testing. Ecol. Monogr. 64, 205-224.

Dornelas, M., Connolly, S.R., Hughes, T.P. 2006. Coral reef diversity refutes the neutral theory of biodiversity. Nature $440,80-82$.

Eberhardt, L.L. 1970. Correlation, regression and density dependence. Ecology 51, 306-310.

Etienne, R.S. 2005. A new sampling formula for neutral biodiversity. Ecol. Lett. 8, 253-260.

Etienne, R.S., Haegeman, B. 2010. The neutral theory of biodiversity with random fission speciation. Theor. Ecol. 'in press'. 
Freckleton, R.P., Watkinson, A.R., Green, R.E., Sutherland, W.J. 2006. Census error and the detection of density dependence. J. Anim. Ecol. 75, 837-851.

Gotelli, N.J., McGill, B.J. 2006. Null versus neutral models: what's the difference? Ecography 29, 793-800.

Haegeman, B., Etienne, R.S. 2010. Self-consistent approach for neutral community models with speciation. Phys. Rev. E 81, 031911.

Halley, J.M. 1996. Ecology, evolution and 1/f-noise. Trends Ecol. Evol. 11, 33-37., doi:10.1016/0169-5347(96)81067-6.

Hanski, I., Foley, P., Hassell, M. 1996. Random walks in a metapopulation: how much density dependence is necessary for long-term persistence? J. Anim. Ecol. 65, 274-282.

Herben, T., Mandák, B., Bímová, K., Münzbergová, Z. 2004 Invasibility and species richness of a community: a neutral model and a survey of published data. Ecology 85, 3223-3233.

Herben, T. 2009. Invasibility of neutral communities. Basic Appl. Ecol. 10, 197-207., doi:10.1016/j.baae.2008.08.006.

Hubbell, S.P. 2001. The unified neutral theory of biodiversity and biogeography. Princeton Univ. Press, Princeton.

Hubbell, S.P., Lake, J.K. 2003. The neutral theory of biodiversity an biogeography, and beyond. In: Blackburn, T.M., Gaston, K.J. (Eds.), Macroecology: Concepts and Consequences. Blackwell, Oxford, pp. 45-63.

Inchausti, P., Halley, J. 2001. Investigating long-term ecological variability using the global population dynamics catabase. Science 293, 655-657.

Inchausti, P., Halley, J. 2002. The long-term temporal variability and spectral colour of animal populations. Evol. Ecol. Res. 4, 1033-1048. 
Keeling, M.J. 2000. Simple stochastic models and their power-law type behaviour. Theor. Popul. Biol. 58, 21-31., doi:10.1006/tpbi.2000.1475.

Kendal, W.S. 2004. Taylor's ecological power law as a consequence of scale invariant exponential dispersion models. Ecol. Complex. 1, 193-209., doi:10.1016/j.ecocom.2004.05.001.

Lande, R., Engen, S., Saether, B.-E. 2006. Stochastic population dynamics in ecology and conservation. Oxford. Univ. Press, Oxford.

Legendre, P., Legendre L. 2003. Numerical Ecology. Elsevier, Amsterdam.

500

Leibold, M.A., McPeek, M.A. 2006. Coexistence of the niche and neutral perspectives in community ecology. Ecology 87, 1399-1410.

Leigh, E.G. 2007. Neutral theory: a historical perspective. J. Evolution. Biol. 20, 2075-2091.

504 McGill, B.J. 2003. A test of the unified neutral theory of biodiversity. Nature 422, $881-885$

McGill, B.J., Maurer, B.A., Weiser, M.D. 2006. Empirical evaluation of neutral theory. Ecology 87, 1411-1423.

Miramontes, O., Rohani, P. 2002. Estimating $1 / \mathrm{f}^{\alpha}$ scaling exponents from short timeseries. Physica D 166, 147-154., doi:10.1016/S0167-2789(02)00429-3.

510 Mitzenmacher, M. 2003. A brief history of generative models for power laws and lognormal distributions. Internet Mathematics 1, 226-251.

512 Murdoch, W.W. 1994. Population regulation in theory and practice. Ecology 75, 271513287.

514 Mutshinda, C.M., O'Hara, R.B.O., Woiwod, P. 2008. Species abundance dynamics 515 under neutral assumptions: a Bayesian approach to the controversy. Funct. Ecol. 22, 340-347. 
Mutshinda, C.M., O’Hara, R.B.O., Woiwod, P. 2009. What drives community dynamics? P. Roy. Soc. B-Biol. Sci. 276, 2923-2929.

Pollard, E., Lakhani, K.H., Rothery, P.1987. The detection of density-dependence from a series of annual censuses. Ecology 68, 2046-2055.

Rosindell, J., Cornell, S.J. 2007. Species-area relationships from a spatially explicit neutral model in an infinite landscape. Ecol. Lett. 10, 586-595.

Rosindell, J., Cornell, S.J. 2009. Species-area curves, neutral models, and longdistance dispersal. Ecology 90, 1743-1750.

Rosindell, J., Cornell, S.J., Hubbbell, S.P., Etienne, R.S. 2010. Protracted speciation revitalizes the neutral theory of biodiversity. Ecol. Lett. 'in press'.

Rosindell, J., Wong, Y., Etienne, R.S. 2008. Coalescence models for spatial neutral ecology. Ecol. Inform. 90, 1743-1750., doi:10.1016/j.ecoinf.2008.05.001.

Shenk, T.M., White, G.C., Burnham, K.P. 1998. Sampling-variance effects on detecting density dependence from temporal trends in natural populations. Ecol. Monogr. 68, 445-463.

Sibly, R.M., Barker, D., Hone, J., Pagel, M. 2007. On the stability of populations of mammals, birds, fish and insects. Ecol. Lett. 10, 970-976.

Taylor, L.R., Woiwod, I.P. 1980. Temporal stability as a density-dependent species characteristic. J. Anim. Ecol. 49, 209-224.

Taylor, L.R., Woiwod, I.P. 1982. Comparative synoptic dynamics. I. Relationships between inter- and intra-specific spatial and temporal variance/mean population parameters. J. Anim. Ecol. 51, 879-906.

Taylor, L.R., Taylor, R.A.J., Woiwod, I.P., Perry, J.N. 1983. Behavioural dynamics. Nature 303, 801-804. 
541 Tilman, D. 2004. Niche tradeoffs, neutrality, and community structure: A stochastic theory of resource competition, invasion, and community assembly. P. Natl. Acad. Sci. USA 101, 10854-10861.

544 Tokeshi, M. 1995. On the mathematical basis of the variance-mean power relationship. Researches on Population Ecology 37, 43-48.

546 Turnbull, L.A., Manley, L., Rees, M. 2005. Niches, rather than neutrality, structure a grassland pioneer guild. P. Roy. Soc. B-Biol. Sci. 272, 1357-1364.

548 Volkov, I., Banavar, J.R., Hubbell, S.P., Martian, A. 2003. Neutral theory and relative 549 species abundance in ecology. Nature 424, 1035-1037.

550 Volkov, I., Banavar, J.R., He, F., Hubbell, S.P., Maritan, A. 2005. Density dependence explains tree species abundance and diversity in tropical forests. Nature 438, 658-661.

554

Volkov, I., Banavar, J.R., Hubbell, S.P., Maritan, A. 2007. Patterns of relative species abundance in rainforests and coral reefs. Nature 450, 45-49.

555 Woiwod, I.P., Hanski, I. 1992. Patterns of density dependence in moths and aphids. J. Anim. Ecol. 61, 619-629. 
Figure legends

559 Figure 1 Schematic illustration of the "archipelago" model. Note that we performed

560 our simulations using much larger numbers of local communities than illustrated here.

561 See the methods for detailed description of the model.

563 Figure 2 Some aspects of real-world populations and those produced by our neutral

564 model. Empirical slopes of Taylor's Power Law and coefficients of determination of TPL of 429 species of aphids, moths and birds were taken from Taylor \& Woiwod $(1980,1982)$. Empirical values of scaling exponent $\gamma$ of power spectra of 544 time series were taken from the Global Population Dynamics Database (GPDD, kindly provided by Pablo Inchausti; see also Inchausti and Halley, 2001, 2002). Box and whisker plots show medians, quartiles and outliers.

Figure 3 Results of the spectral analysis of population time series produced by a neutral model. $\gamma$ is the scaling exponent in $S(f) \propto 1 / f^{\gamma}$ where $S(f)$ is spectral density and $f$ is frequency. Displayed are the effects of the neutral model's parameters (Tab.

574 1) on $\gamma$ as a box and whisker plot with median and quartiles. A random walk of 575 sufficient length would have $\gamma=2$.

$577 \quad$ Figure 4 Results of the testing for density dependence in population time series 578 produced by neutral model as a box and whisker plot with median and quartiles.

579 Pollard's $P$ is the probability of obtaining the data in case of density independence

580 (Eq. 1; Pollard et al., 1987). 
582 Figure 5 The slopes of temporal Taylor's power law (TPL) as produced by neutral

583 models with various levels of model parameters. Solid horizontal lines delimit the

584 range observed in natural populations. Shown as a box and whisker plot with median

585 and quartiles shown, outliers are not displayed.

586

587 Figure 6 Dependence of Pollard's $P$ on relative abundance of species in local

588 communities and in metacommunities. Shades of grey represent density of points in

589 hexagonal bins.

590

591 Figure 7 Dependence of slope and coefficient of determination $\left(\mathrm{R}^{2}\right)$ of Taylor's

592 Power Law on mean relative abundance of species in local communities. Shades of

593 grey represent density of points in hexagonal bins. 


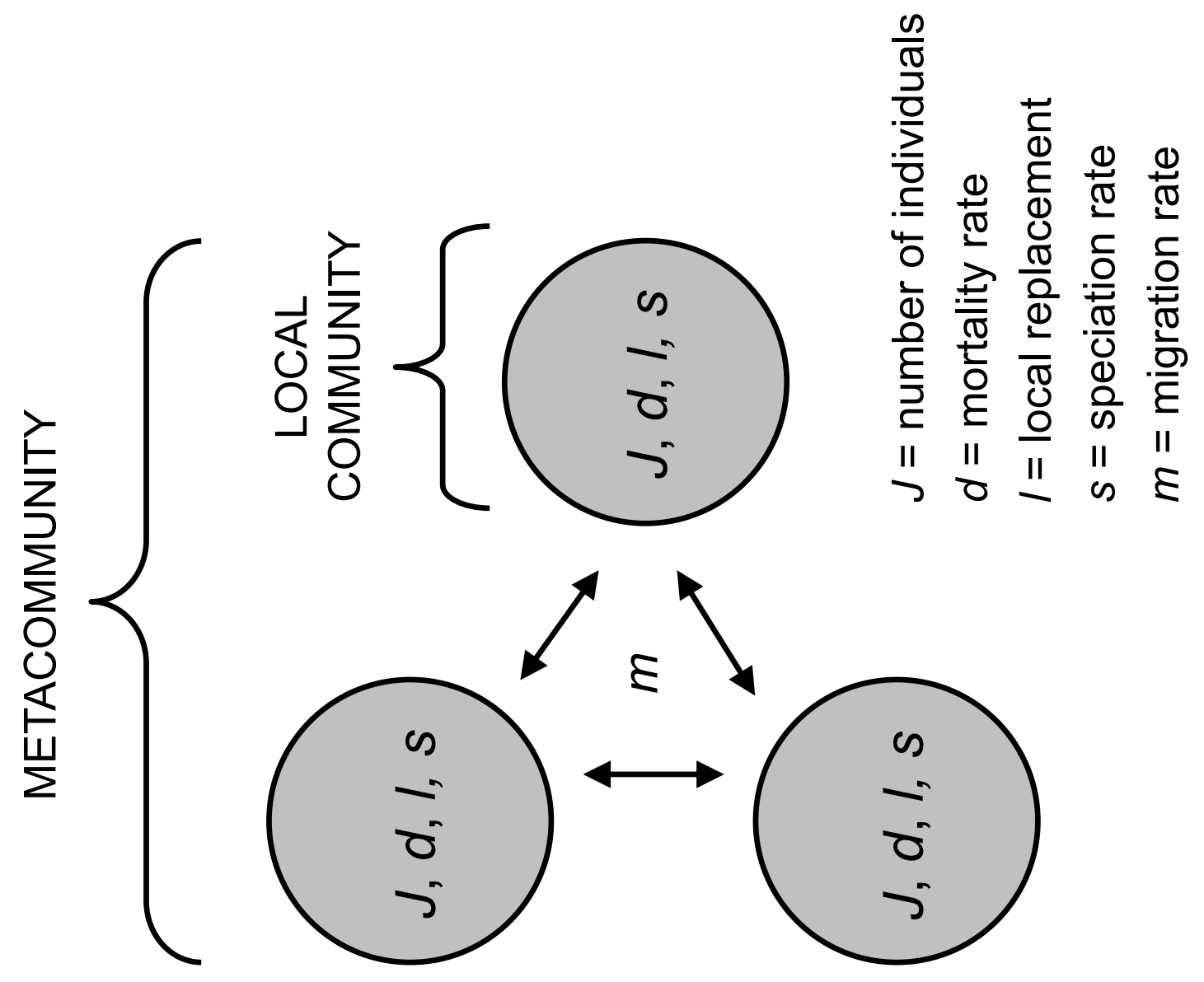



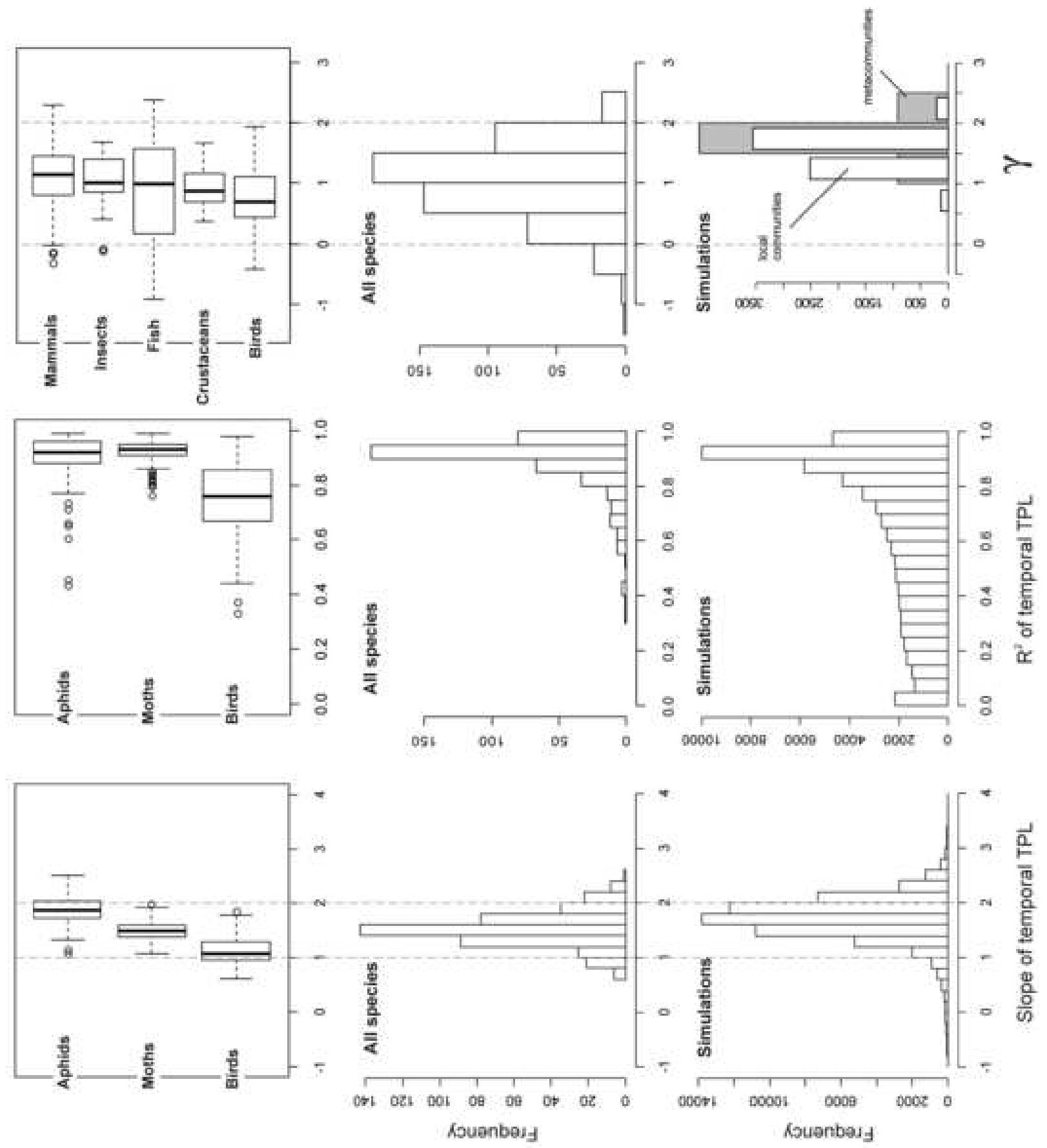


\section{Figure}

LOCAL COMMUNITIES
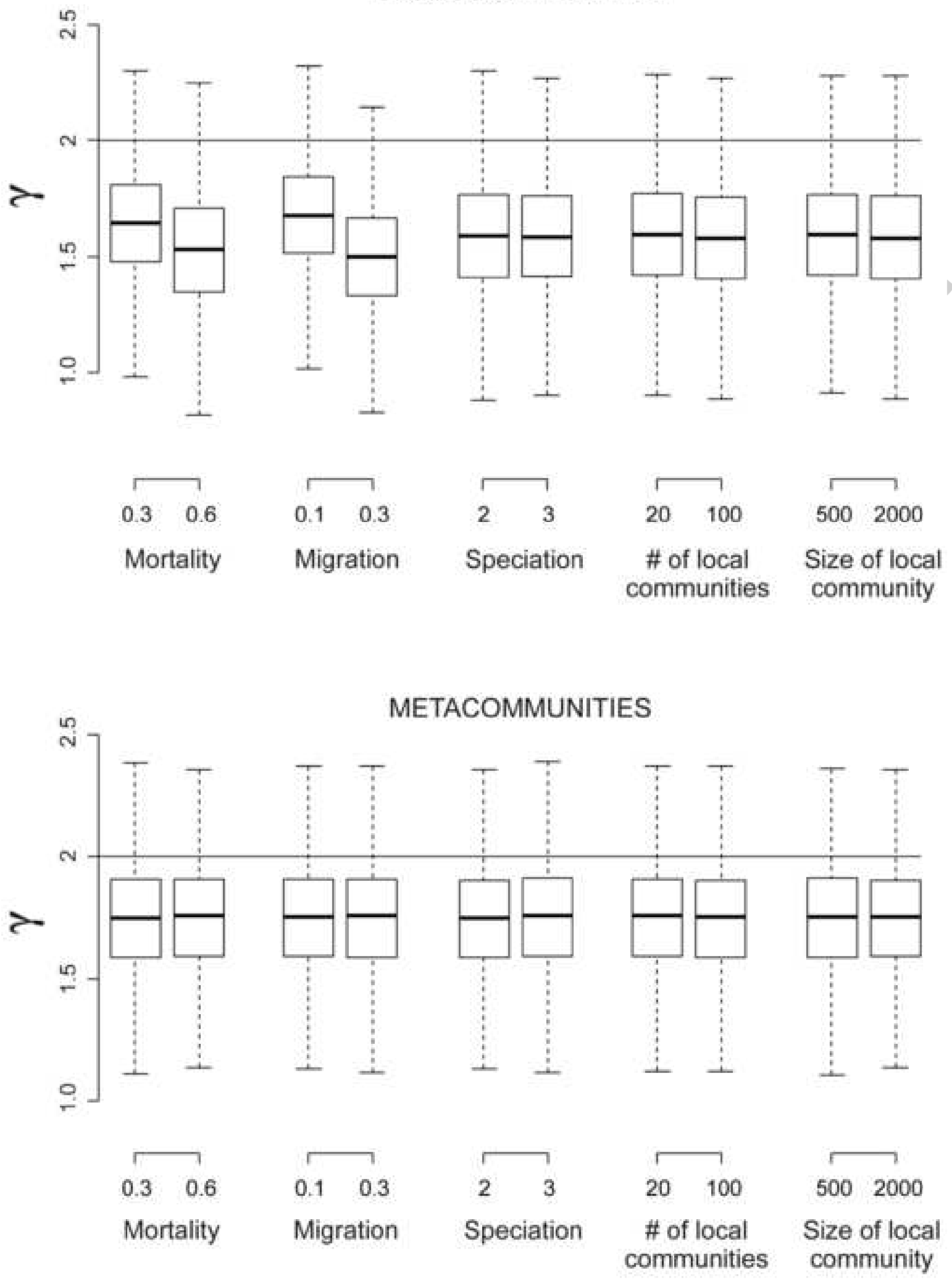


\section{Figure}

LOCAL COMMUNITIES
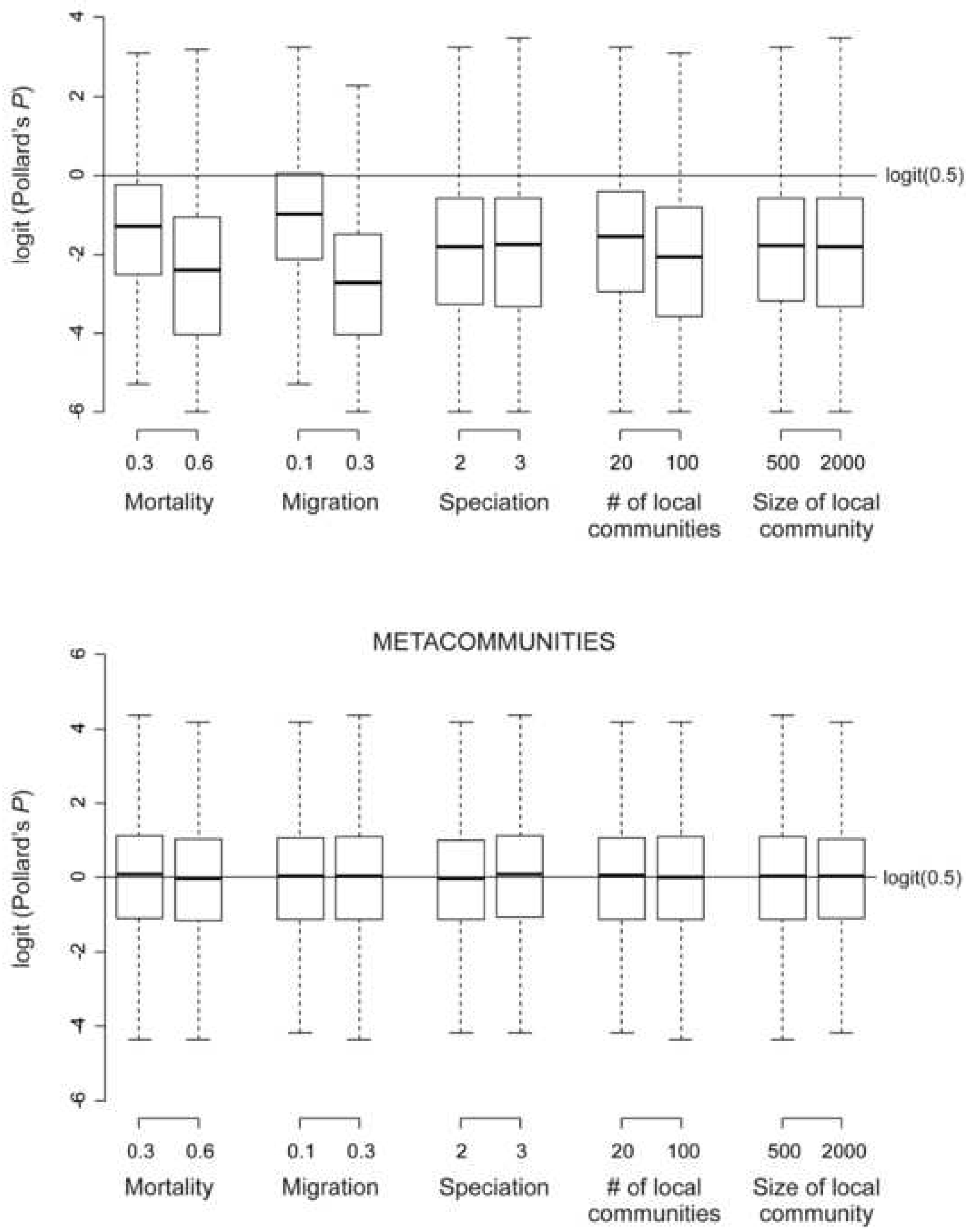


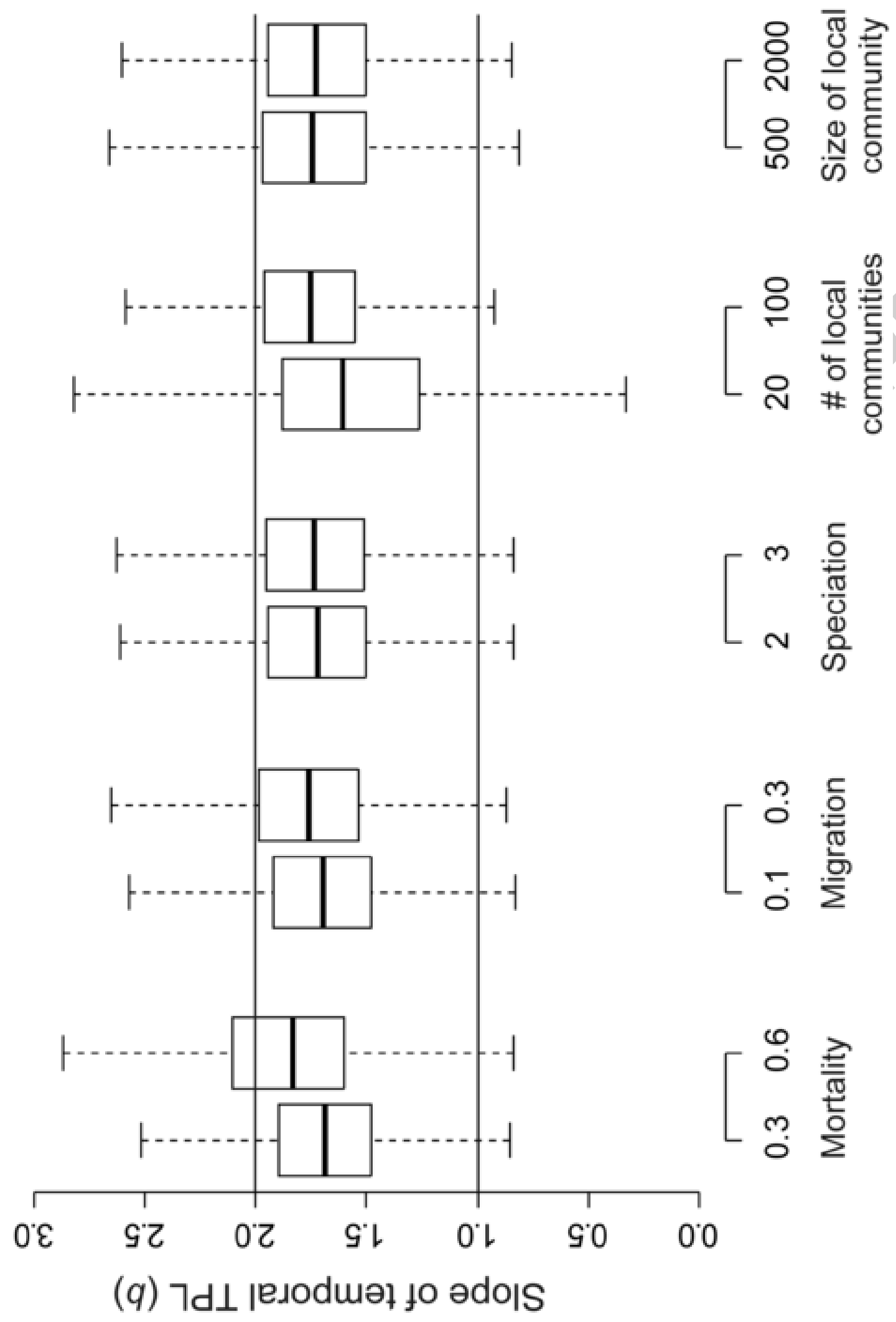




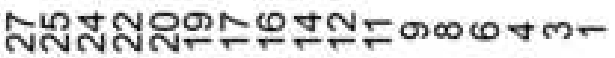

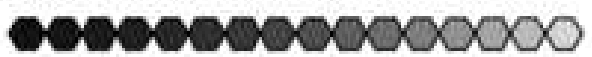

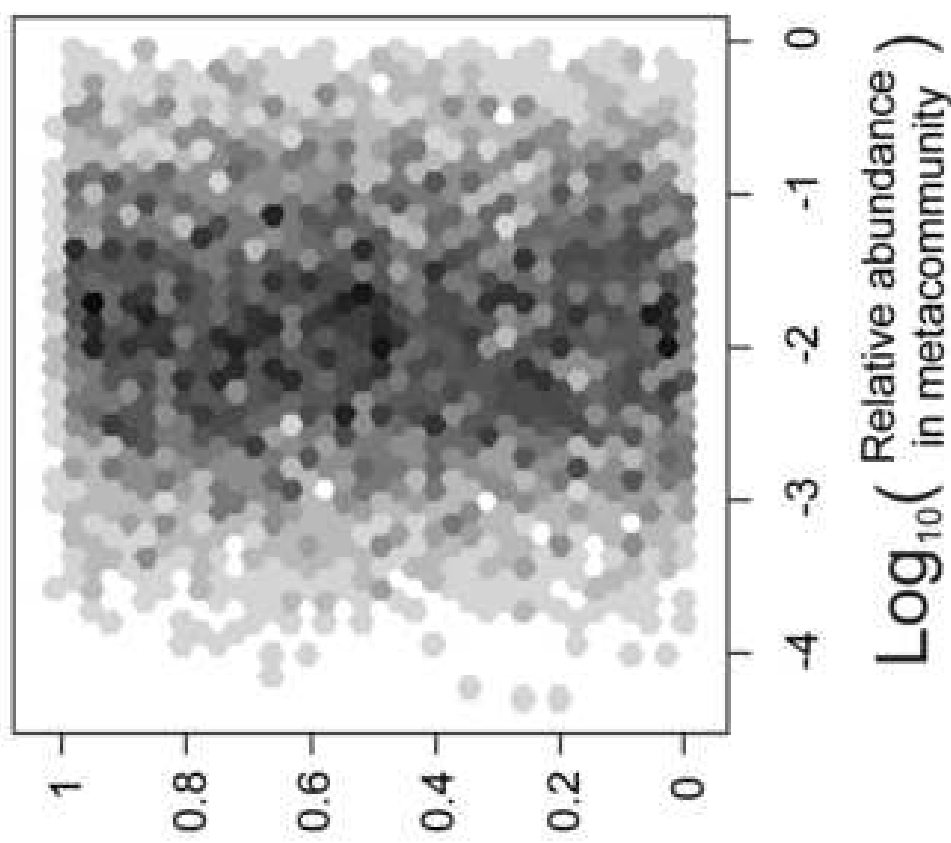

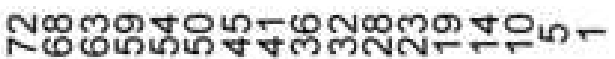

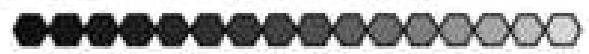

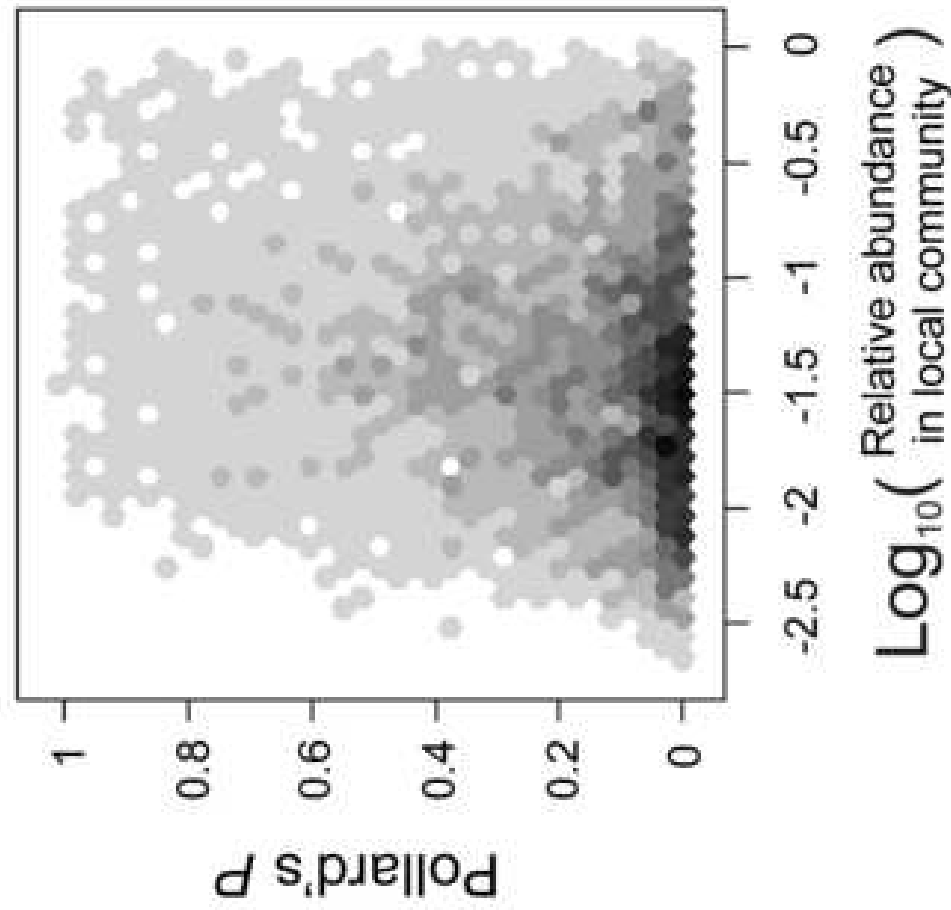




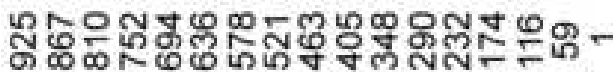

ceeverenO00000

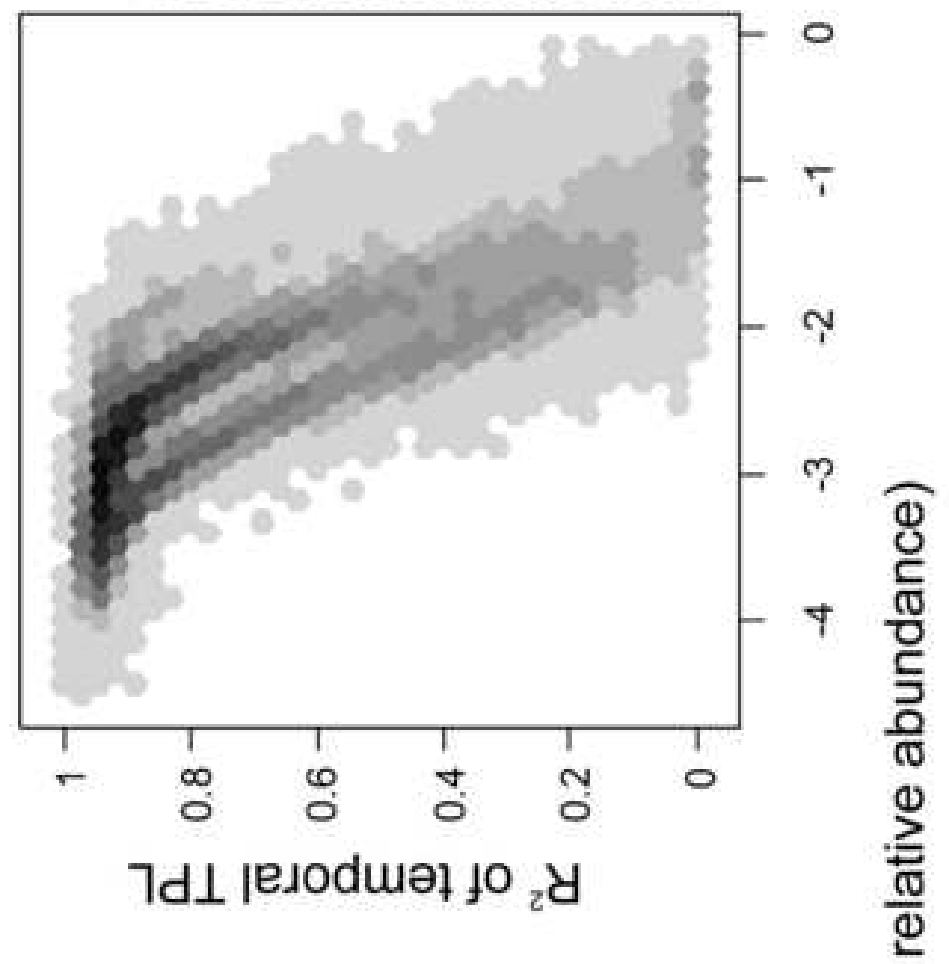

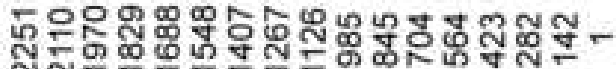

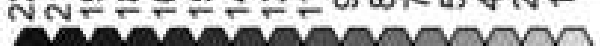
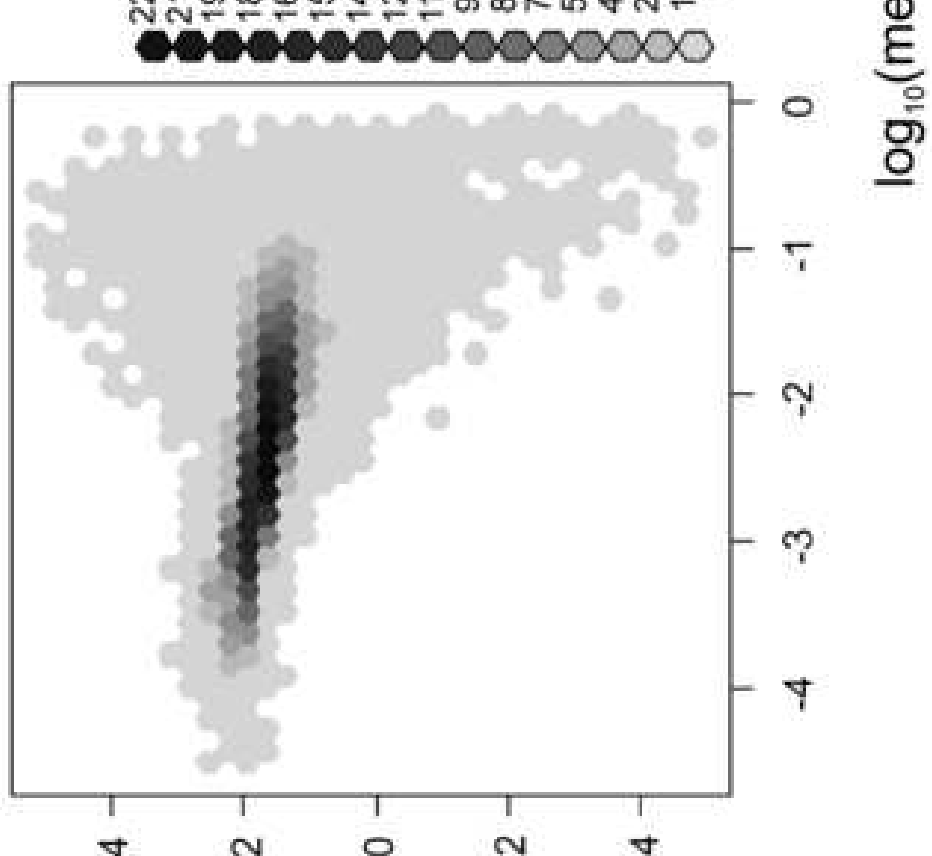

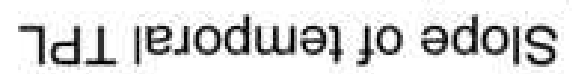


Table 1 An overview of the parameters and their levels used in our neutral model simulations. See material and methods for detailed description of the exact function of each parameter.

\begin{tabular}{lll}
\hline Parameter & Description & Levels used in \\
& & simulations \\
\hline Number of local & number of local communities that make up & 100,20 \\
communities & the metacommunity & 2000,500 \\
Size of each local & number of individuals in each local & \\
Mortality rate $(d)$ & mortality rate of individuals per time step in & $0.6,0.3$ \\
& the local community & $0.1,0.3$ \\
Migration rate $(m)$ & proportion of vacant positions in the & \\
& community replaced by migration from other & \\
& local communities & $2 \times 10^{-6}, 3 \times 10^{-6}$ \\
Speciation rate $(s)$ & speciation rate per species per step &
\end{tabular}


Table 2 The strength of the effect of each parameter on the value of $\gamma$ and the logittransformed Pollard's $P$. Parameter $\gamma$ describes the 'colour' of the spectra of a time series. Pollard's $P$ is the probability of obtaining the data in case of density independence (random walk). The values of $\mathrm{R}^{2}$ were estimated using a multiple ANOVA. Figures in bold show values higher than 0.001 .

\begin{tabular}{lccccc}
\hline & \multicolumn{3}{c}{ Local communities } & \multicolumn{3}{l}{ Metacommunities } \\
& $\mathrm{R}^{2}(\gamma)$ & $\mathrm{R}^{2}$ (logit of & $\mathrm{R}^{2}(\gamma)$ & $\mathrm{R}^{2}$ (logit of & $\mathrm{R}^{2}$ (slope of \\
& & Pollard's $P$ ) & & Pollard's $P$ ) & $\mathrm{TPL}$ ) \\
& & & & & \\
\hline Mortality rate & $\mathbf{0 . 0 4 5}$ & $\mathbf{0 . 0 7 8}$ & $<0.001$ & $<0.001$ & $\mathbf{0 . 0 1 9}$ \\
Migration rate & $\mathbf{0 . 1 1}$ & $\mathbf{0 . 1 9}$ & $<0.001$ & $<0.001$ & $\mathbf{0 . 0 0 1 4}$ \\
Speciation rate & $<0.001$ & $<0.001$ & $<0.001$ & $<0.001$ & $<0.001$ \\
\# of local & $<0.001$ & 0.014 & $<0.001$ & $<0.001$ & $\mathbf{0 . 0 4 8}$ \\
communities & & & & & \\
Size of local & $<0.001$ & $<0.001$ & $<0.001$ & $<0.001$ & $\mathbf{0 . 0 0 1 5}$ \\
community & & & & & \\
\hline
\end{tabular}

